\title{
Tracheal ring fracture and early tracheomalacia following percutaneous dilatational tracheostomy Eu Chin $\mathrm{Ho}^{1}$, Atul Kapila*2 and William Colquhoun-Flannery ${ }^{1}$
}

Address: ${ }^{1}$ Department of Otolaryngology, Royal Berkshire Hospital, Reading, Berkshire, RG1 5AN, UK and ${ }^{2}$ Department of Anaesthetics, Royal Berkshire Hospital, Reading, Berkshire, RG1 5AN, UK

Email: Eu Chin Ho - euchinho@yahoo.co.uk; Atul Kapila* - atul.kapila@rbbh-tr.nhs.uk; William Colquhoun-Flannery - will.colquhounflannery@rbbh-tr.nhs.uk

* Corresponding author

Published: 31 August 2005

BMC Ear, Nose and Throat Disorders 2005, 5:6 doi:10.1 186/1472-68/5-5-6

This article is available from: http://www.biomedcentral.com/1472-68/5/5/6

(c) 2005 Ho et al; licensee BioMed Central Ltd.

This is an Open Access article distributed under the terms of the Creative Commons Attribution License (http://creativecommons.org/licenses/by/2.0), which permits unrestricted use, distribution, and reproduction in any medium, provided the original work is properly cited.
Received: 14 January 2005

Accepted: 31 August 2005

\begin{abstract}
Background: Percutaneous dilatational tracheostomy (PDT) is increasingly popular within intensive care units for patients who need prolonged ventilatory support. Significant complications are rare.

Case presentation: Our patient suffered tracheal ring fracture and early tracheomalacia following this procedure. These complications are demonstrated in our accompanying video.

Conclusion: Contrary to common beliefs, tracheal rings are commonly fractured during the PDT procedure. The consequent granulation can lead to tracheal stenosis and tracheomalacia.
\end{abstract}

\section{Background}

In many intensive care units within the United Kingdom, the Percutaneous Dilatational Tracheostomy (PDT) technique had replaced the traditional open (surgical) tracheostomy method for patients who need prolonged ventilatory support. This technique is often performed by the intensivists themselves, hence is time and cost effective. 2 complications of this technique are described and shown in a video accompanying this report.

\section{Case presentation}

Our patient was a 78 year old lady who following a total hip replacement developed a pneumothorax and pneumonia and needed post-operative ventilation. She was difficult to wean off the ventilator and on day 7 post-op, we decided to perform a combined open and percutaneous dilatational tracheostomy (PDT). Due to the difficult anatomy, we dissected down to the level of the strep muscles before proceeding with PDT technique. The tracheal was easily palpated by this stage. We used a Portex ULTRAperc (Portex Ltd, Hythe, Kent, England) kit, with a size 8.0 $\mathrm{mm}$ ID tracheostomy tube. Her tracheal rings were rather calcified and we heard a 'crack' during the dilatation process. Nevertheless, there was no problem with introducing the tracheal tube. The procedure was carried out under guiding vision from within the trachea by flexible video bronchoscopy with no obvious complications noted.

Subsequently this lady failed three attempts at tracheal decannulation. She would develop immediate airway obstruction following tracheostomy tube removal requiring reinsertion of the tracheostomy tube. On day 32 of her ICU stay, following the third attempt, we performed a video endoscopic airway examination. Passing the flexible bronchoscope (Pentax FB-18BS, Pentax Medical, Slough, England) through the larynx, we found a fractured tracheal cartilage ring, protruding into the trachea just above the tracheostomy tube. Then, by passing the 


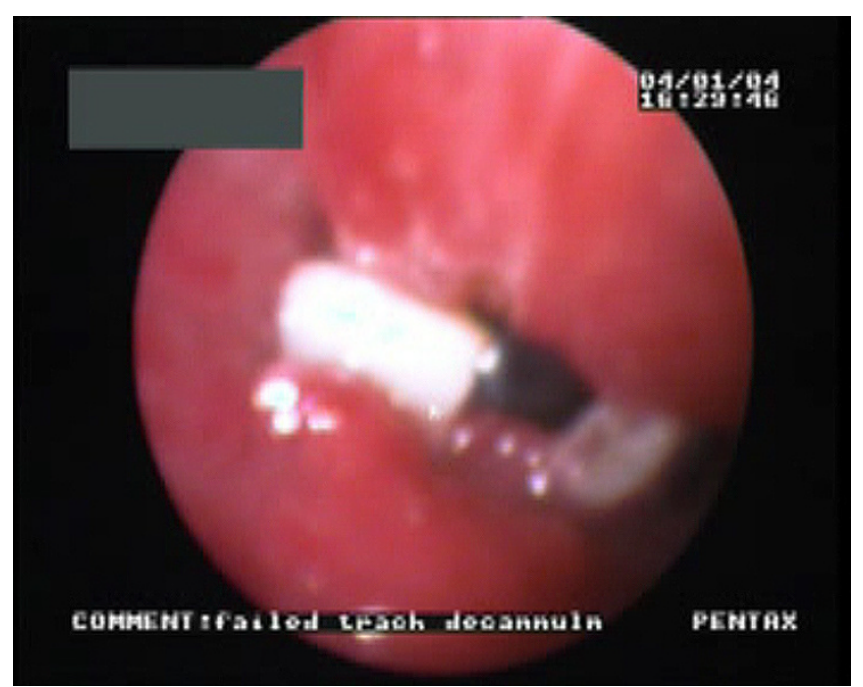

Figure I

Fractured tracheal ring prolapsing into tracheal lumen above tracheostomy tube.

flexible bronchoscope through the tracheostomy tube, we saw tracheal wall oedema and inflammation. There was also collapse of the anterior tracheal wall on inspiration demonstrating tracheomalacia. This segment of tracheal wall collapse was quite long as shown by the partial removal of the tracheostomy tube.. [Figure 1 \& see video file 1]

She was eventually discharged to a ward environment on day 38 with the tracheostomy tube in situ. Unfortunately she later suffered a cardio-respiratory arrest and the patient died. The post mortem findings were consistent with that of respiratory failure secondary to bronchopneumonia.

\section{Discussion}

Our patient had a tracheal ring fracture and early distal tracheomalacia following a PDT procedure. It is our opinion that the tracheal ring fracture would have been caused during the PDT procedure, either by the dilator or by the tracheostomy tube itself. Why did the bronchoscopist not spot this at the time of the procedure? As the fractured tracheal ring was sitting above the tracheostomy tube, it was possible that the in-situ translaryngeal endotracheal tube at the time of the procedure could have prevented displacement of the fractured fragments. Even though video bronchoscopy guidance is virtually routine nowadays, perhaps it is not $100 \%$ failsafe.

It is often believed that tracheal rings are displaced but remain intact with the PDT technique. In one study, PDT was performed on cadaveric specimens, which demonstrated significant peristomal mucosal tear and cartilaginous fracture with this technique [1]. Well calcified tracheal rings would have made this complication more likely. The consequent granulation and scarring can lead to tracheal stenosis at the subglottic, suprastomal or stomal level $[1,2]$. Stenosis at a suprastomal level is believed to be caused by anterior tracheal wall injury and granulation formation from the tracheostomy procedure and tube itself [2].

Autopsies of patients who had tracheostomy also showed necrosis of the tracheal cartilage and mucosal ulceration beyond the tracheostomy site as early as 2 weeks post cannulation [3]. Together with the irritating effects of the tracheostomy cuff, these changes are likely to be responsible for the distal tracheomalacia seen in our patient. Other possible causes of tracheomalacia would include the use of oversized cannula and infection. However, it is important to keep things in perspective, as these complications are rare.

We tried to obtain consent for publishing this case report from the patient's next of kin. However, all attempts to contact the patient's family were unsuccessful.

\section{Conclusion}

Contrary to common beliefs, tracheal rings are commonly fractured during the PDT procedure. The consequent granulation can lead to tracheal stenosis and tracheomalacia. Nevertheless, by understanding the pathophysiology behind the complications, these risks could be minimised.

\section{Competing interests}

The author(s) declare that they have no competing interests.

\section{Authors' contributions}

ECH was overall coordinator and drafted, revised and background researched the paper.

AK made the video recording of the complications and critically revised the article.

WC supervised the project and critically revised the article. 


\section{Additional material}

\section{Additional File 1}

Video demonstrating the 2 complications in this patient. Firstly, the video demonstrated the fractured tracheal ring prolapsing into tracheal lumen. This was recorded by passing a fiberoptic bronchoscope per nasally. Later in the video, distal tracheomalacia was demonstrated by passing the fiberoptic bronchoscope through and partially withdrawing the tracheostomy tube.

Click here for file

[http://www.biomedcentral.com/content/supplementary/14726815-5-6-S1.mpg]

\section{References}

I. Hotchkiss KS, McCaffrey JC: Laryngotracheal injury after percutaneous dilatational tracheostomy in cadaver specimens. Laryngoscope 2003, I I3:16-20.

2. Koitschev A, Graumueller S, Zenner HP, Dommerich S, Simon C: Tracheal stenosis and obliteration above the tracheostoma after percutaneous dilatational tracheostomy. Critical Care Medicine 2003, 31:1574-1576.

3. van Heurn LWE, Theunissen PHMH, Ramsay G, Brink PRG: Pathologic Changes of the Trachea after Percutaneous Dilatational Tracheotomy. Chest 1996, 109:1466-1469.

\section{Pre-publication history}

The pre-publication history for this paper can be accessed here:

http://www.biomedcentral.com/1472-6815/5/6/prepub

Publish with Biomed Central and every scientist can read your work free of charge

"BioMed Central will be the most significant development for disseminating the results of biomedical research in our lifetime. "

Sir Paul Nurse, Cancer Research UK

Your research papers will be:

- available free of charge to the entire biomedical community

- peer reviewed and published immediately upon acceptance

- cited in PubMed and archived on PubMed Central

- yours - you keep the copyright

Submit your manuscript here:

http://www.biomedcentral.com/info/publishing_adv.asp 